\title{
The end of the road for darbepoetin alfa in heart failure?
}

T

he results of the RED-HF trial have been reported in the New England

Journal of Medicine. In the RED-HF study, clinical outcomes were compared for the erythropoiesis-stimulating agent (ESA) darbepoetin alfa and placebo in patients with chronic heart failure (HF) and mild-to-moderate anaemia not due to iron deficiency. Although treatment with darbepoetin alfa was associated with an early and sustained increase in haemoglobin levels, the therapy was not associated with a reduced risk of deathor hospitalization for $\mathrm{HF}$, the primary outcome of the trial. "We believe that these neutral findings are robust, given the long-term follow-up and large number of events in our study," says Professor Karl Swedberg, first author on the RED-HF trial report.

Anaemia and iron deficiency are common in patients with HF and, although iron deficiency is often the cause of anaemia in these patients, the two conditions do not necessarily co-exist. Dr John Nanas (University of Athens, Greece)-who was not involved in the RED-HF trial, but has researched the importance of anaemia and iron deficiency in patients with HF-explains that the association between anaemia and adverse outcomes in patients with $\mathrm{HF}$ had resulted in the hypothesis that anaemia correction might improve the prognosis of these patients. Previous studies have suggested that anaemia treatment, using an ESA, might improve functional capacity and reduce hospitalization in patients with chronic $\mathrm{HF}$ and anaemia. However, in their trial report, the RED-HF investigators point out that this evidence comes from small studies and is not robust.

The RED-HF investigators, therefore, set out to test whether darbepoetin alfa affects clinical outcomes in patients with systolic HF (NYHA functional class II-IV and left ventricular ejection fraction $\leq 40 \%$ ) and moderate anaemia (haemoglobin level 9.0-12.0 g/dl), but no iron deficiency (transferrin saturation $\geq 15 \%$ ) in a large, multinational, randomized, controlled trial. All patients were also receiving guidelinerecommended therapy for HF. Patients' blood iron levels were assessed every 3 months during the trial and, if transferrin saturation was determined to be $<20 \%$ at any time, patients were administered iron supplements.

In total, 1,136 patients were randomly allocated to receive darbepoetin alfa and 1,142 patients were assigned to receive placebo. Median follow-up was 28 months. In the patients receiving the ESA, the median haemoglobin level increased from $11.2 \mathrm{~g} / \mathrm{dl}$ at baseline to $13.0 \mathrm{~g} / \mathrm{dl}$ at the end of follow-up. By contrast, in the placebo group, the median haemoglobin levels at baseline and end of follow-up were determined to be $11.2 \mathrm{~g} / \mathrm{dl}$ and $11.5 \mathrm{~g} / \mathrm{dl}$, respectively. The difference between the two groups was statistically significant by 1 month and remained significant throughout the study.

Death from any cause or first hospitalization from worsening HF occurred in $50.7 \%$ of patients receiving darbepoetin alfa and $49.5 \%$ of patients taking placebo (HR 1.01, 95\% CI 0.90$1.13, P=0.87)$. The lack of a darbepoetinalfa-associated effect on the primary outcome was consistently observed for all subgroups assessed. Death from any cause and death from cardiovascular causes did not differ between the two groups (HR 1.04, 95\% CI 0.92-1.19, $P=0.51$, and HR 1.04, 95\% CI 0.91-1.20, $P=0.56$, respectively). Darbepoetin alfa also had no effect on first hospitalization for worsening $\mathrm{HF}$ (HR 0.99, 95\% CI $0.85-1.16, P=0.92)$. Notably, embolic and thrombotic events occurred more often with darbepoetin alfa (13.5\% vs $10.0 \%$ with placebo, $P=0.009$ ).

"Our findings do not support the use of an ESA in patients with systolic HF and a haemoglobin concentration between $9.0 \mathrm{~g} / \mathrm{dl}$ and $12.0 \mathrm{~g} / \mathrm{dl}$ ", says Professor Swedberg. In their trial report, the RED-HF investigators concluded that their findings "suggest that the haemoglobin level, like other surrogates, is simply a marker of poor prognosis in $\mathrm{HF}$, rather than a therapeutic target." They acknowledge, however, that they "tested one approach to elevating haemoglobin levels, and other strategies may have different effects."

Indeed, not all researchers are ready to give up on the idea of treating anemia in patients with HF. "The failure of up-to-date studies to demonstrate a significant association between long-term prognosis and anaemia correction could be attributable to the characteristics of the selected population," says Dr Nanas. He believes that investigators still need "to define the most appropriate therapy and to identify a chronic HF patient subpopulation that might benefit from anemia correction".

Dr Nanas comments that it would be "intriguing to investigate, in a population similar to the patients enrolled in RED-HF, whether anaemia would have responded to iron replenishment alone and whether this would have led to an improvement in outcome." Professor Swedberg also highlights that the importance of iron deficiency in HF, and the effects of iron replacement, remain to be explored.

Bryony M. Mearns

Original article Swedberg, K. et al. Treatment of anemia with darbepoetin alfa in systolic heart failure (RED-HF). N. Engl. J. Med. doi:10.1056/NEJMoa1214865

Further reading van Veldhuisen et al. Anemia and iron deficiency in heart failure: mechanisms and therapeutic approaches. Nat. Rev. Cardiol. 8, 485-493 (2011) 